\title{
Qualitative systematic review of barriers and facilitators to patient-involved antipsychotic prescribing
}

Rebecca Pedley, Caitlin McWilliams, Karina Lovell, Helen Brooks, Kelly Rushton, Richard J. Drake, Barnaby Rumbold, Vicky Bell and Penny Bee

\section{Background}

Despite policy and practice mandates for patient involvement, people with serious mental illness often feel marginalised in decisions about antipsychotic medication.

\section{Aims}

To examine stakeholder perspectives of barriers and facilitators to involving people with serious mental illness in antipsychotic prescribing decisions.

\section{Method}

Systematic thematic synthesis

\section{Results}

Synthesis of 29 studies identified the following key influences on involvement: patient's capability, desire and expectation for involvement, organisational context, and the consultation setting and processes.

\section{Conclusions}

Optimal patient involvement in antipsychotic decisions demands that individual and contextual barriers are addressed. There was divergence in perceived barriers to involvement identified by patients and prescribers. For example, patients felt that lack of time in consultations was a barrier to involvement, something seldom raised by prescribers, who identified organisational barriers. Patients must understand their rights to involvement and the value of their expertise. Organisational initiatives should mandate prescriber responsibility to overcome barriers to involvement.

\section{Declaration of interest}

None.

\section{Copyright and usage}

(C) The Royal College of Psychiatrists 2018. This is an Open Access article, distributed under the terms of the Creative Commons Attribution-NonCommercial-NoDerivatives licence (http:// creativecommons.org/licenses/by-nc-nd/4.0/), which permits non-commercial re-use, distribution, and reproduction in any medium, provided the original work is unaltered and is properly cited. The written permission of Cambridge University Press must be obtained for commercial re-use or in order to create a derivative work.
In recent decades, there has been a philosophical shift in healthcare whereby patients have been recognised not just as passive recipients of care, but as experts in their own condition, who hold a right to be involved in treatment decisions. ${ }^{1,2}$ Shared decision-making $(\mathrm{SDM})$ in healthcare is a practice where patient and provider expertise are brought together (i.e. through information exchange) to reach agreement on treatment decisions. ${ }^{3,4}$ Recognition of the importance of SDM has been driven by calls from the consumer rights movement to reject traditional paternalistic models of care and uphold patients' ethical and moral right to self-determination. ${ }^{4}$ Proponents of SDM argue that its practise enables better decisionmaking and ultimately, improved clinical outcomes. ${ }^{5}$

The practice of SDM is now widely enshrined in policy as a key facet of patient-centred care, ${ }^{6,7}$ yet many patients in mental health settings report that it is yet to be routinely adopted. ${ }^{8,9}$ Although people with mental health conditions frequently express a preference to be involved in their care, ${ }^{10}$ it has been argued that, in comparison with physical health conditions, the practice of SDM might be inhibited by the unique challenges associated with mental health conditions. In particular, the ability for practitioners to legally enforce care against the patient's wishes may act as a significant threat to SDM that inhibits the development of a collaborative partnership. ${ }^{11}$ The concept of 'capacity' in mental health poses as a further obstacle, which may reduce the practitioners' willingness to implement SDM. ${ }^{12}$

SDM may be particularly warranted in the prescribing of antipsychotic medication, the mainstay of treatment for people with serious mental illness (SMI). ${ }^{13}$ Most antipsychotics have comparable efficacy ${ }^{14}$ and, aside from clozapine, similar effectiveness. ${ }^{15-17}$ However, adverse effect profiles vary and there are appreciable differences in the risks of stigmatising effects such as tardive dyskinesia and those affecting physical health, for example, the risk of diabetes, atherosclerosis and sudden cardiac death. ${ }^{18}$ Consequently, there is an argument for basing treatment decisions not only on effectiveness, but on side-effect burden. It seems important that those affected by side-effects should be involved in these decisions.

There is a clear need to understand why patient involvement in antipsychotic prescribing decisions does not take place. To our knowledge, no systematic review has sought to understand the barriers and facilitators to patient involvement in antipsychotic prescribing. To address this gap, the present study aims to systematically review published qualitative evidence on the barriers and facilitators to involving patients with SMI in antipsychotic prescribing decisions. Given the paucity of evidence examining SDM interventions for people with mental health conditions, ${ }^{19}$ a synthesis of evidence of barriers and facilitators from key stakeholder perspectives (patient, carer and health professional) may yield insight into how future interventions could address the issue.

\section{Method}

\section{Systematic search strategy}

Electronic database searches of Ovid Medline, Embase, PsycINFO and CINAHL (Cumulative Index to Nursing and Allied Health Literature) were conducted from database inception to June 2016. Search terms were generated by team discussion and a review of the literature for four concepts; (a) patient, carer or health professional perspectives, (b) collaborative involvement, (c) antipsychotic medication, and (d) qualitative design. The concepts were combined 
and applied (the full search strategy is detailed in supplementary Table DS1 available online at http://doi.org/10.1192/bjo.2017.5) and a validity test was conducted using a set of four test papers known to be relevant to the review aim.

Search results were exported to EndNote, duplicates deleted and the remainder imported to Covidence (www.covidence.org), a webbased software product to assist the screening and organisation of systematic reviews. Titles and abstracts were independently screened against the eligibility criteria (Table 1) by two researchers, with conflicts resolved via third party agreement. Full texts for all potentially eligible papers were obtained. For texts that were published in abstract form only, targeted author searches were conducted to identify additional full text publications by the same author (one additional paper was identified using this method). ${ }^{20}$ Full texts were independently screened by two researchers, with conflicts referred to a third author for resolution.

\section{Critical appraisal and data extraction}

Quality indicators were integrated from a variety of guidelines, ${ }^{21-23}$ and included whether the studies reported the methodology, selection of participants, data collection method and analysis method. No study was excluded on quality alone. Data extraction of quality indicators and descriptive study characteristics, such as country of origin and participant demographics, was undertaken by one of four authors and checked by a second reviewer. Two authors extracted study outcomes independently, and discrepancies were resolved by discussion.

\section{Synthesis}

An inductive thematic synthesis approach ${ }^{24}$ was conducted collaboratively by two authors (R.P. and C.M.), with regular consultation with wider team members. Figure 1 provides a detailed breakdown of the process. Primary findings were labelled as free codes according to (a) meaning and (b) participant perspective (i.e. patient, health professional or carer). We employed the constant comparative method to ensure translation of concepts from one study to another. ${ }^{24,25}$ This involved looking for the similarities and differences between papers and comparing new codes with our existing framework. Descriptive themes emerged during this stage to describe groups of codes which shared common meaning or experience. As the synthesis progressed, it became evident that some groups of codes would be better subsumed into larger themes, and other groups arose as distinct from others. The final stage of synthesis involved 'going beyond' the primary data, and has been described as the defining characteristic of synthesis. ${ }^{26}$ For this stage, we drew parallels between various descriptive categories and began to generate a new interpretation, not explicitly presented in the primary findings. Particular barriers and facilitators were considered in terms of which contexts and which stakeholder perspective they emerged from.

\section{Results}

The search resulted in 29 studies reported across 30 included papers for synthesis; $;^{20,27-55}$ the flow of studies is outlined in Fig. 2. All study characteristics are detailed in the supplementary material (Tables DS2 and DS3). Of the 30 studies, 13 were conducted in the $\mathrm{UK}^{27,28,30,33,36,37,41,44,46,47,49,51-53}$ ten in the USA, ${ }^{20,29,31,32,34,38,39,43,48,50}$ four in Canada, ${ }^{35,40,42,55}$ and one each in Norway ${ }^{45}$ and Sweden ${ }^{54}$. Sixteen studies examined data from only patients' perspectives, ${ }^{28,29 \text {, }}$ $32,33,37,39-43,45,46,49,50,53,55$ six from only health professionals' perspectives, ${ }^{30,34,35,47,51,52,54}$ and only one from carers' perspectives. ${ }^{36}$ The six remaining studies examined a combination of stakeholder perspectives, ${ }^{27,31,38,44}$ two of these including carer viewpoints. $^{20,48}$ This resulted in 886 participants; 647 patients, 215 health professionals and 24 carers.

Of the 22 studies that included patients, ${ }^{20,27-29,31-33,37-46,48-50,53,55}$ seven included participants with diagnosis of psychosis/ schizophrenia, ${ }^{38,42,45,46,49,53,55}$ one involved participants with a diagnosis of bipolar ${ }^{50}$ and one included participants with dual diagnoses. ${ }^{20}$ Nine studies had a sample of mixed diagnoses, ${ }^{29,32,33,37,39,41,43,44,48}$ and four described their sample as having 'SMI' but did not provide any further details on diagnosis. ${ }^{27,28,31,40}$

Of the 12 studies that included health professionals, ${ }^{20,27,30 \text {, }}$ $31,34,35,38,44,48,51,52,54$ seven recruited a mixed sample of professionals such as psychiatrists, nurses, case managers and peer support specialists, ${ }^{20,27,31,34,35,38,44}$ three recruited only psychiatrists, ${ }^{30,47,51,52}$ one recruited only nurses ${ }^{54}$ and one recruited only case managers. $^{48}$

Sixteen studies recruited participants from a community care setting, ${ }^{20,28,30-34,36-38,42,46,48,50,51,54,55}$ five from mixed settings, ${ }^{27,29,35,39,47}$

\section{Table 1 Inclusion/exclusion criteria}

Inclusion criteria

Peer review journal articles or conference papers, reported in English.

Qualitative methodology. Data collected by interview, focus group or ethnography and analysed using thematic type analysis method as a minimum.

Data must be primary findings reporting the views of stakeholders Where participants are patients, at least $75 \%$ of the sample has a primary diagnosis of SMI. Where participants are health professionals/family or carers, at least $75 \%$ care for at least one person with SMI.

SMI population meeting one of the following: (a) defined as 'SMI'; (b) diagnosed with psychosis, schizophrenia or related disorders, chronic and/or recurrent major depression, personality disorder or bipolar disorder, or (c) patients in secondary care mental health services who are prescribed antipsychotics.

Note- where criterion a or b of the population criteria is met, any medication use will be operationalised as antipsychotic use.

Reports barriers/facilitators to the involvement of patients in antipsychotic prescribing decisions.

\section{Exclusion criteria}

Unpublished studies (e.g. dissertations), abstracts, single case studies. Quantitative data or data collected in written format, e.g. open-ended questionnaire. No attempt to analyse data thematically. Researchers did not directly seek stakeholder perspectives, e.g. observational data collected from a prescribing interaction.

Data are not primary findings from stakeholder perspectives.

Patients without a primary diagnosis of SMI.

Participants are health professionals or carers/family members caring for individuals without primary diagnosis of SMI.

SMI population not explicitly specified.

No barriers/facilitators to patient involvement in antipsychotic prescribing decisions reported. No author interpretation of findings relating to research question. 


\begin{tabular}{|c|c|c|c|}
\hline \multirow[b]{2}{*}{ 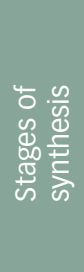 } & Open coding & $\begin{array}{c}\text { Grouping into 'descriptive } \\
\text { themes' }\end{array}$ & Generating 'analytical themes' \\
\hline & $\begin{array}{l}\text { Two authors interpreted each extracted } \\
\text { data point and agreed on a code. Codes } \\
\text { were labelled by the stakeholder group } \\
\text { from which they had originated. At this } \\
\text { stage, coding stayed close to the data. }\end{array}$ & $\begin{array}{l}\text { Through discussion, codes were grouped } \\
\text { together with similar codes that shared } \\
\text { common meaning or experience } \\
\text { (descriptive themes). Barriers and } \\
\text { facilitators were retained separately } \\
\text { within each descriptive theme. }\end{array}$ & $\begin{array}{l}\text { Descriptive themes were discussed within } \\
\text { the wider team. Deeper analytical } \\
\text { interpretation was drawn through } \\
\text { comparison of themes and with reference to } \\
\text { points of similarity and differences between } \\
\text { stakeholder groups. }\end{array}$ \\
\hline 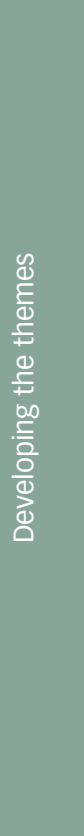 & $\begin{array}{l}\text { Example codes: } \\
\text {-Time restraints (P) } \\
\text {-Insurance companies overturning } \\
\text { decisions (HP) } \\
\text {-Trust psychiatrist to make the choice (P) } \\
\text {-P emphasised need to respect their own } \\
\text { expertise (P) } \\
\text {-P felt perceived as malingerers, time } \\
\text { wasters, not taken seriously (P) } \\
\text {-P reluctance to question authority (P) } \\
\text {-Provision of information (P) } \\
\text {-Lack-of-capacity barrier to discussing side } \\
\text {-effects (HP) } \\
\text {-Important to elicit P preferences (HP) } \\
\text {-Software led to disclosure of previously } \\
\text { undisclosed material (HP) }\end{array}$ & $\begin{array}{l}\text { Descriptive themes (white, bold) } \\
\text { with example codes (black italics) } \\
\quad \text { Attitudes and Beliefs } \\
\text {-Trust psychiatrist to make the choice } \\
\text { (P, barrier) } \\
\text {-P emphasised need to respect their own } \\
\text { expertise (P, facilitator) } \\
\text { - Skills and knowledge } \\
\text {-Provision of information (P, facilitator) } \\
\text {-Lack of capacity barrier to discussing side- } \\
\text { effects (HP, barrier) } \\
\quad \text { Context } \\
\text {-Time restraints (P, barrier) } \\
\text {-Insurance companies overturning } \\
\text { decisions (HP, barrier) } \\
\text { - Staff-patient relations } \\
\text {-P felt perceived as malingerers, time } \\
\text { wasters, not taken seriously (P, barrier) } \\
\text {-P reluctance to question authority } \\
\text { (P, barrier) } \\
\quad \text { In-consultation processes } \\
\text {-Important to elicit P preferences } \\
\text { (HP, facilitator) } \\
\text {-Software led to disclosure of previously } \\
\text { undisclosed material (HP, facilitator) }\end{array}$ & $\begin{array}{l}\text { Analytical theme titles: } \\
\text { - Expectations and desire for collaborative } \\
\text { prescribing } \\
\text { - Perceptions of patient capability for } \\
\text { involvement } \\
\text { - Serious mental illness as a barrier } \\
\text { - Patient knowledge and } \\
\text { capability } \\
\text { - The organisational context } \\
\text { - The consultation setting and processes } \\
\text { affecting involvement } \\
\text { - The consultation setting } \\
\text { - Process factors } \\
\text { - Tools and support aids }\end{array}$ \\
\hline
\end{tabular}

Fig. 1 Diagram showing the development of analytical themes. Further analysis at the descriptive stage led to the understanding that the setting and nature of the interaction with health professionals ('The consultation setting and processes affecting involvement') formed the central theme, which was bi-directionally related to the remaining themes. HP, health professional; P, patient.

one study each from an in-patient forensics ward, ${ }^{53}$ primary care setting ${ }^{44}$ and the readership of an alternative health magazine, ${ }^{40}$ and five studies did not specify service setting. ${ }^{41,43,45,49,52}$

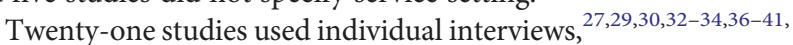
$43,45-47,49,51-55$ six used focus groups ${ }^{20,31,35,42,44,50}$ and two used a mixture of both. ${ }^{28,48}$ Twelve studies used thematic analysis ${ }^{27,30,32,34,37,39,43,45,47-49,51,54}$ and five used grounded theory. ${ }^{28,38,41,46,53}$ Seven used another type of analysis, e.g. content analysis (non-quantitative type), ${ }^{20,29,33,35,40,52,55}$ while the remaining five studies did not specify the analysis method ${ }^{31,36,42,44,50}$ but were deemed to have used a thematic type analysis by the study team.

A table presenting study quality indicators is presented in the supplementary material (DS4). No study was judged to meet all quality indicators. All studies met at least one criterion, with 20 out of 29 studies meeting at least half the criteria ( $\geq 4$ out of the eight quality criteria met). The most reliably met quality indicator was the method of data collection, reported by all 29 studies.

\section{Data synthesis}

Figure 1 shows how the analysis progressed from descriptive themes to a more developed interpretation that went 'beyond' the original study findings. As the descriptive themes were compared and contrasted, it was recognised that the nature and setting of events within the consultation lay at the centre of the influencing factors. This led to further refinement of themes; for example, upon further consideration, it was noted that the context theme consisted of two core components: firstly, the nature of the consultation setting and, secondly, those factors that originated from the organisational context. As such, the first component was transposed to the new 'central' theme, alongside the processes taking place within the consultation.

\section{Emerging themes}

Five themes emerged from the analysis, which can be subsumed under two broader themes, the underlying influences on patient involvement and the influences on involvement specific to the consultation. Each broad theme and its sub-themes will now be described.

\section{The underlying influences on involvement}

Three underlying influences on patient involvement were identified: perceptions of patient capability for involvement, the organisational context, and expectations and desire for collaborative prescribing.

\section{Perceptions of patient capability for involvement}

Serious mental illness as a barrier. Concerns about the negative impact of patients' mental health problems on their capability for involvement emerged as a prominent theme. Health professionals, patients and carers agreed that there were times (particularly during 'crisis' periods) when the active symptoms of mental illness, cognitive impairment and lack of insight prevented patients from being meaningfully involved in medication decisions. ${ }^{20,27,30,35,41,46-48,50,52,53}$ Despite patients' doubts about 


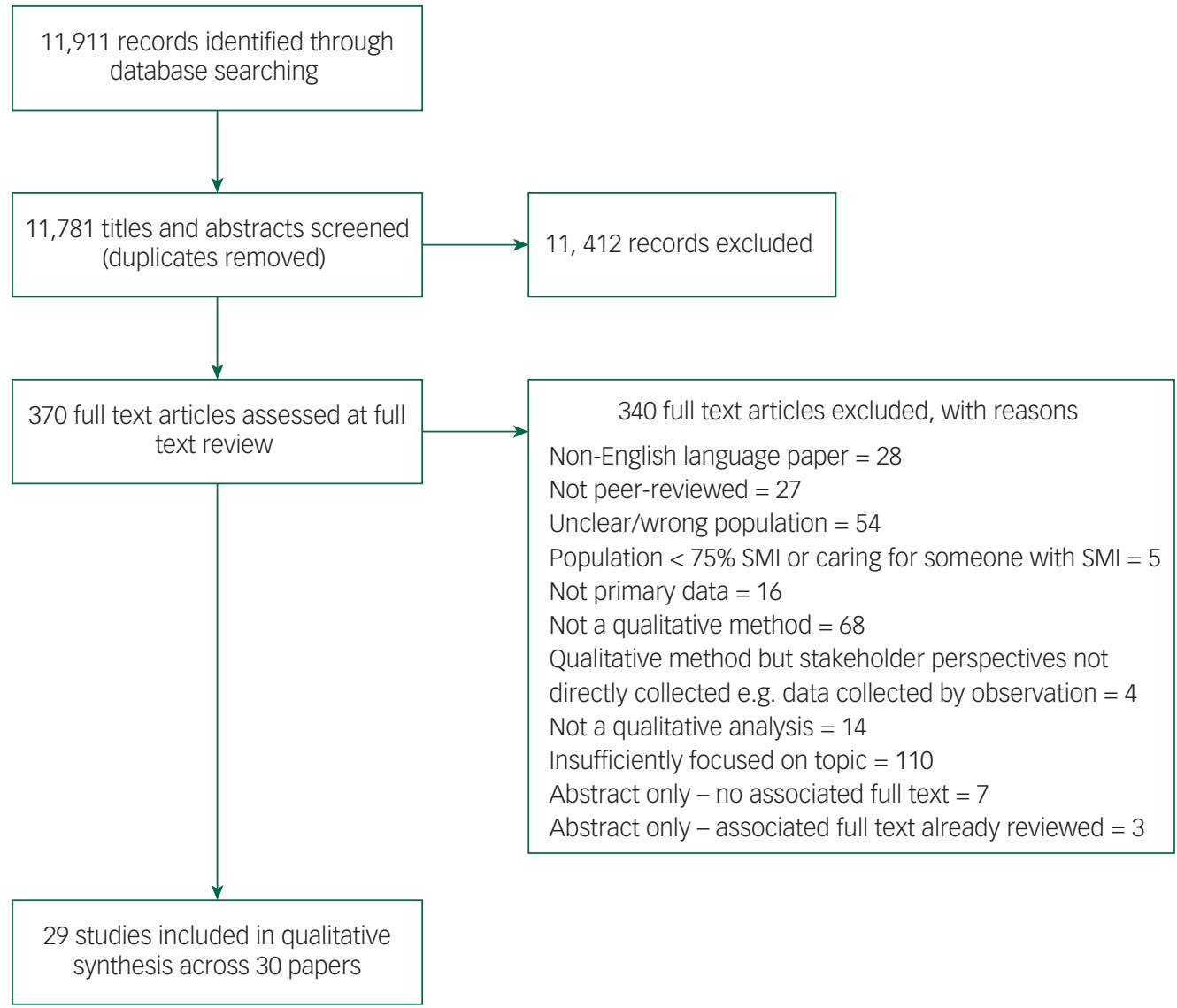

Fig. 2 PRISMA flowchart. SMI, serious mental illness.

their own capacity for involvement when severely unwell, they did not necessarily think it right for them to be excluded from decisions outside these times. Some patients felt that receiving a diagnosis of a mental illness could lead health professionals to 'assume a certain level of incompetency' (p. 32 in Mahone et $\mathrm{al}^{20}$ ), suggesting that judgements of patients' capability for involvement may be based on more generalised expectations of low capability, irrespective of capacity. Consistent with this, case managers taking part in one study perceived patients to lack key capabilities for involvement, e.g. in knowing how to research drugs. ${ }^{48}$ In one study, individual differences in psychiatrists' attitudes to capacity were apparent, with some attempting to maximise involvement despite lack of capacity (including attempts to understand the patient's wishes prior to being unwell), and others regarding its lack as an 'absolute contraindication' to patient involvement. ${ }^{52}$ While there may be consensus between stakeholders that a degree of mental well-being and capacity are needed for involvement, precisely where that threshold lies appears to be more subjective and based on individual attitudes.

Patients' knowledge and confidence. Patients believed that the receipt of high-quality medication information was an important requisite for involvement. They widely reported having not been provided with the necessary knowledge to make medication decisions, including the range of drug options (e.g. depot rather than tablet medication) or the side-effects, the purpose, or even the name of their prescribed medication. ${ }^{28,33,42,43,45,46,48}$ At times, poor understanding related to patients receiving low-quality information (e.g. medication leaflets), but this could also result from simply receiving no information, or only receiving information when it was too late, (e.g. being told about weight gain following increased weight). ${ }^{43}$ Patients felt that being given information of a higher quality and quantity could lead to increased involvement, not only through increasing their knowledge, but also by enhancing their confidence and levels of empowerment. ${ }^{20,32,34,37,39,44,48}$ The importance of patients' confidence was highlighted in one study of young adults with SMI, which indicated that involvement increased through gaining knowledge and by having a prescriber who encouraged involvement. ${ }^{32}$ Confidence was also increased through successful prior experiences of involvement, achieving accomplishments, gaining control over life in general or simply through growing older. ${ }^{32}$

Expressions of concern about patients having inadequate knowledge about medication were notably less prominent within health professional perspectives. In one study, although professionals acknowledged patients' entitlement to honest information, they also discussed the need to limit, or even deliberately withhold certain information about side-effects. This was to avoid patients becoming confused, as well as to ensure the best possible clinical outcome, e.g. telling a young person about the risk of weight gain may discourage them from taking the medication. ${ }^{48}$ This raises the prospect that patients sometimes receive inadequate information owing to health professionals' concern about their ability to use this information responsibly.

\section{The organisational context}

Pressure on prescribers from within the healthcare context was a clear, though less widely discussed, influence on the level of patient involvement. Prescribers working with patients in forensic settings spoke of the need to select a drug that would manage 
behaviour, 'even if all the antipsychotic does is sedate them' (p. 6 in Shepherd $e t a l^{52}$ ). Pressure from other staff, including nurses, who may push prescribers to select a drug they have 'faith in', could also restrict opportunities for involvement. ${ }^{52}$

The wider organisational context was seen by health professionals as restricting their flexibility in prescribing. Resource limitations and restrictions imposed by funding bodies (statutory services or insurance companies) were regarded by health professionals as determining the choice of medication, in some cases restricting the opportunity to review decisions altogether. ${ }^{20,52}$ For example, health professionals in one study were unwilling to review medication decisions once a patient was sufficiently well to be discharged from in-patient settings owing to the pressure to free beds. ${ }^{52}$ This was also highlighted by carers, who perceived a reluctance to prescribe 'newer' drugs as a possible consequence of their cost. ${ }^{36}$ Professional and legal responsibilities enacted to ensure the safety and well-being of the patient and members of the public, including safeguarding against harm, ${ }^{20}$ were perceived to further hinder collaborative prescribing. Family members shared this concern, questioning 'how far can he compromise his recommendation and still be a responsible physician?' (p. 31 in Mahone et $a l^{20}$ ). Health professionals in one study felt that the growth of patient and consumer groups had encouraged their practice to move towards more patient-centred practice and, as such, was a facilitator to collaborative prescribing. ${ }^{51}$

\section{Expectations and desire for collaborative prescribing}

Findings within this theme indicated that for collaborative prescribing to be a success, patients and prescribers need to value both their own and their partner's contribution to the process. Patients and prescribers agreed that both parties needed to possess the openness and willingness to allow patient involvement to take place. ${ }^{20,28,48}$ However, stakeholder views showed that not all patients expect or want an active role in medication decisions, with some opting to take a passive approach, relying solely on the expertise of the prescriber. ${ }^{27,46,50,53,55}$ Evidence highlighted a number of factors contributing to passivity, including patients' belief in the superior value of professionals' opinions ${ }^{50}$ and being unaware of their own entitlement to contribute, ${ }^{49}$ as well as past experiences of services where involvement had been denied..$^{20,32,54}$ The latter contributing factor included patients who had received services during adolescence (where professionals and parents made the choices) or who had been exposed to extended periods of care within a traditional medical model. ${ }^{20,32,54}$ As one participant stated: '... you've been making this decision for me for so long, why start now ...' (p. 31 in Mahone et $\left.a l^{20}\right)$. Patients and health professionals noted the need to empower patients as vital. ${ }^{48}$

Not all patients were in doubt about the importance of their contribution to the prescribing process, with some identifying the need to value their own expertise. ${ }^{20}$ A contrasting minor theme within health professional perspectives indicated that although passivity posed a challenge to SDM, patients holding inflexible views about medication could be equally disruptive. Fixed views could take the form of reluctance to consider any medication ${ }^{20}$ or, in the case of a study involving patients with personality disorder, an insistence on being prescribed medication despite a perceived lack of clinical justification. ${ }^{47}$ In such cases, rather than depending solely on the health professionals' opinion, patients failed to value the expertise of the prescriber.

\section{The consultation setting and processes affecting involvement}

The factors affecting involvement described so far illustrate the underlying contextual, attitudinal and belief-based underlying factors within the proposed model (Fig. 3) that underpin collaborative prescribing. A central theme within the data, where a large proportion of the study findings clustered, related to the consultation setting, as well as the processes and actions that take place within it, which directly help or hinder the collaborative process.

\section{The consultation setting}

The degree to which patients could be involved in prescribing was dependent on an environment that was conducive to collaborative discussion. Patients complained that their input in prescribing decisions was restricted by a lack of practical opportunity for discussion. Consultations were experienced as an 'in and out' process in which prescribers appeared visibly 'bothered' and rushed. ${ }^{32,43,48,53}$ Patients indicated that longer consultations allowed greater involvement, as these had allowed patients 'time to go through it properly'. ${ }^{33,44}$ Unavailability of time was rarely raised as a barrier to patient involvement by health professionals, the exception being a study conducted within a primary care setting. ${ }^{44}$ However, health professionals in another study indicated the importance of not appearing rushed (i.e. remaining calm) and in giving patients adequate time to process and respond to information. ${ }^{30,51}$

Patients in one study reported that involvement opportunities were restricted by the infrequency of appointments, which health professionals were reluctant to increase, or which were precluded by external factors (relating back to the organisational context theme) such as insurance restrictions. ${ }^{32}$ There was some evidence that individual psychiatrist attitudes and willingness to ensure their accessibility (e.g. provision of their direct phone number) could help to overcome such barriers. ${ }^{32}$

Lack of continuity of care posed a further barrier to involvement, with patients' attempts to gain input further obstructed by interim health professionals: 'you'll have to talk when you get a regular doctor' (p. 74 in Lacasse et $\mathrm{l}^{43}$ ). In a study where a supplementary pharmacist prescriber had been trialled, greater continuity of care gave participants improved opportunity for discussion. ${ }^{33}$ Another factor that affected patients' ease when entering prescribing discussions was the number of people present; too many attendees could make patients feel overwhelmed. ${ }^{27}$

\section{Process factors}

Relational factors. A significant factor influencing patient involvement in antipsychotic decisions was the behaviours of those involved in the prescribing discussion, with responsibility placed particularly on the health professional. The need for health professionals to convey their positive regard towards the patient was key, with health professionals highlighting the need to display empathy, warmth and respect in their interactions. ${ }^{30,51}$ These qualities were not always evident to patients, some of whom felt negatively perceived by health professionals (e.g. as 'malingerers' or 'time wasters'), ${ }^{20,44}$ disrespected and treated with little compassion, ${ }^{43}$ e.g. 'do you want to be fat, or do you want to be crazy?' (p. 75 in Lacasse et al ${ }^{43}$ ). This patient perception was supported by one study where some staff held negative stereotypes of patients as unreliable, uneducated and potentially violent. ${ }^{44} \mathrm{~A}$ widely reported barrier to involvement was when patients' views were 'brushed off ${ }^{20,32,37,38,40,45,53}$ or, more rarely, overtly rejected by the health professional, e.g. when under a court order. ${ }^{43}$ Conversely, patients described the behaviour of health professionals who encouraged involvement as those who listened and responded (e.g. by acting upon side-effect information) and who demonstrated openness and the willingness to take their views seriously (e.g. by not minimising concerns). ${ }^{29,32,33,39,45}$ One strategy that health professionals raised as demonstrating interest involved taking down notes about patients' lives (e.g. the names of their children) so that such details could be 


\section{UNDERLYING INFLUENCES ON INVOLVEMENT}

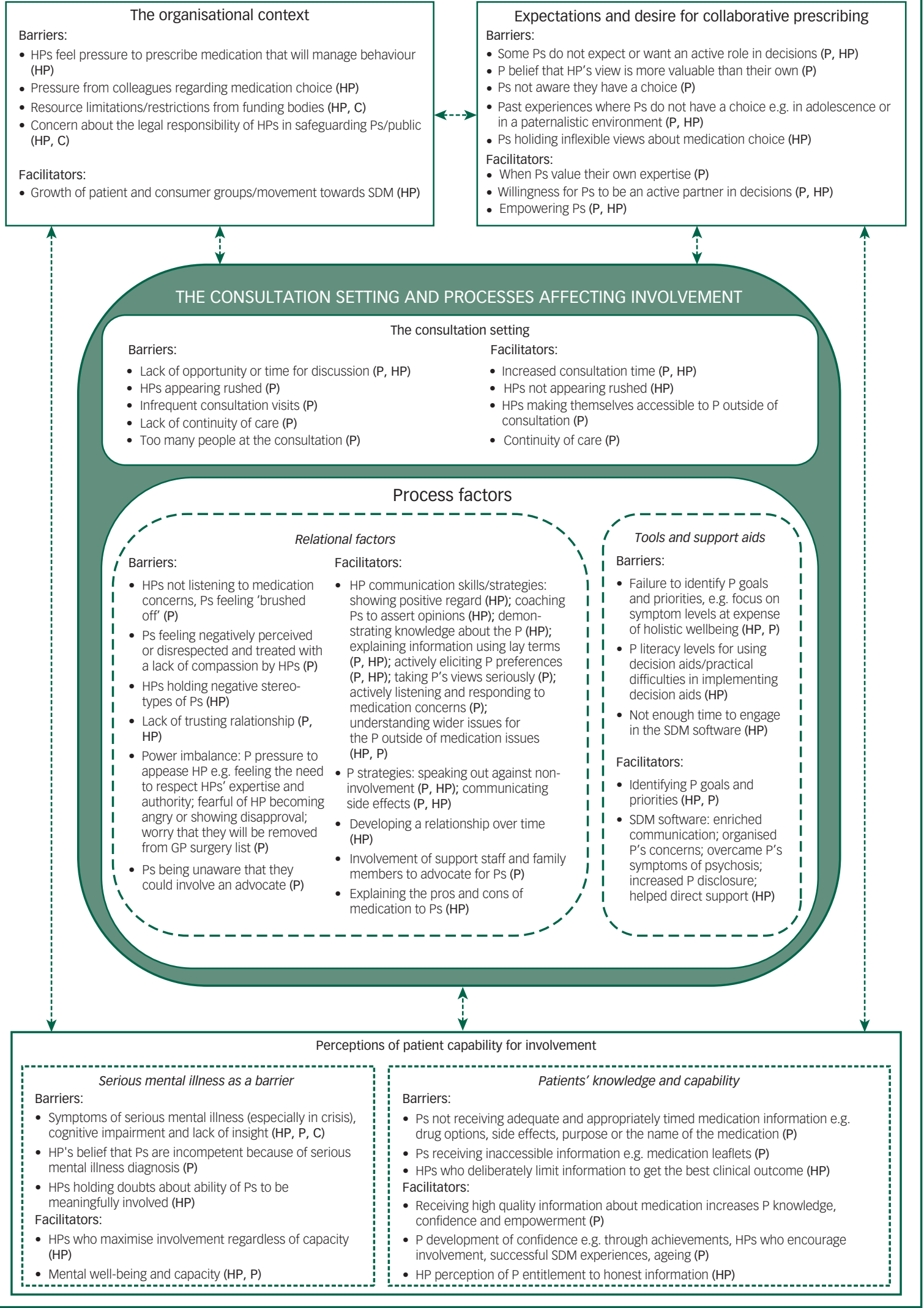

Fig. 3 Diagram of patient involvement. C, carer; HP, health professional; P, patient; SDM, shared decision-making. (P, HP, C) indicates which stakeholder's perspective this is from. 
recounted during subsequent consultations to give the impression of a 'personal service'.

There was also some evidence that patients held negative views of staff, with some speaking of being unable to trust health professionals, eroding their ability to be open and honest about their medication. ${ }^{43}$ Health professionals acknowledged that lack of patient trust could act as a barrier to involvement, which they attributed to their power to enforce mental health law. ${ }^{52}$ Further evidence of this power imbalance was evident in the way some patients acted to appease the prescriber. Sometimes, this was due to a belief in the need to respect the medical practitioners' expertise and authority. ${ }^{43,48}$ Patients felt a pressure to 'please' the provider, 'keep the peace' and allow the meeting to progress quickly. ${ }^{20,32,48}$ At other times, open dialogue was inhibited by a fear of the prescriber becoming angry or showing disapproval, or by patients' fear of being perceived as difficult or even manipulative. ${ }^{20,43,44,48}$ Less frequently, fears were attributed to the powers that health professionals held to bring serious negative consequences to patients, such as being removed from the general practitioner surgery list. ${ }^{44}$ In response to this, non-prescribing nurses interviewed in one study actively coached some patients to assert opinions when meeting with the prescriber, for example, by working with them to develop their courage. ${ }^{54}$

Stakeholders perceived health professionals' communication as key to the success of collaborative prescribing. Professionals needed to take time in explaining relevant information about medication in non-technical, person-centred language, and actively elicit preferences around medication. ${ }^{20,30,48,51,52}$ Health professionals recognised that their ability to demonstrate such skills may require additional training, for example, in motivational interviewing. ${ }^{20}$ It was also considered important that discussions extended beyond medication to broader aspects of the patients' lives, including their illness, background, views and the underlying reasons for how they felt and behaved. ${ }^{20,30,32,43,44}$ While communication was important in 'getting to know' the patient, some health professionals emphasised the need to develop this relationship over time. ${ }^{30}$

Although most findings focused on the health professionals' role in facilitating patient involvement, there was some recognition of the patient's part in instigating change, by speaking out against decisions made without their involvement and by communicating side-effects to their prescriber. ${ }^{20,28,45}$ Health professionals validated these facilitators, emphasising the need for patients to 'speak up' and be 'reliable communicators'. ${ }^{48}$

Another means of facilitating patient communication was through the involvement of non-prescribing staff members and family members who could advocate for patients, helping to overcome barriers such as low confidence or dismissive prescriber attitudes. ${ }^{32,38,44}$ It was recognised, however, that some patients were unaware that they could involve an advocate, indicating that some individuals may be denied such support. ${ }^{27}$ The need to strengthen patients' capability for involvement was recognised by non-prescribing nurses who, when working with patients they perceived to be cooperative, actively sought to enhance their ability in medication decisions, by explaining the pros and cons of medications. ${ }^{54}$

Tools and support aids. Evidence from a number of studies suggested that collaborative discussions could be aided by the use of tools and support aids, which helped to improve the quality of discussions and ensured patients' perspectives were heard. One such strategy was the identification of patient priorities and goal setting. Studies evaluating experiences of using an SDM computer program found that this software enabled the identification of patient priorities, leading to increased involvement. ${ }^{31,38}$ It was notable that where meaningful goals had not been identified or where a shared understanding of patient priorities had not been developed, involvement was less likely. ${ }^{28,32,38}$ This is exemplified when health professionals focus solely on symptom levels, rather than taking a broader, holistic view of overall well-being. ${ }^{28,32}$

Patients and health professionals found that SDM packages encouraged conversation ${ }^{38}$ and ensured that patients' concerns were quickly recognised and addressed. ${ }^{31}$ Health professionals believed that this was of particular benefit to patients whose mental health problems interfered with their communication skills. ${ }^{31}$ Such electronic packages were also found to increase patients' disclosure, with health professionals reporting that patients found it 'easier to tell the computer'. ${ }^{31,38}$ For example, use of the package led one mother to reveal fears that taking her sedating medication would cause her to sleep through alerts from her child's sleep apnoea monitor. ${ }^{31}$ This understanding enabled health professionals to direct support to this concern. ${ }^{31}$

A small number of highly specific barriers to the implementation of shared decision aids were raised. These included the difficulties for patients with literacy problems in using decision aids, ${ }^{20}$ difficulties in ensuring that patients arrived promptly enough to make use of the software package, ${ }^{31}$ and the challenge for staff in working with the large amounts of information generated by the software tool. ${ }^{31}$

\section{Discussion}

Our synthesis has led to the identification of a number of key factors that affect patients' involvement. Within the hypothesised model (Fig. 3), three factors outside the consultation - (a) patients' capability, (b) the organisational context, and (c) the expectation and desire for collaborative prescribing - determine the consultation setting and processes affecting involvement. Stakeholders' experiences of the consultation setting and processes also feed back into the three factors outside the consultation, forming a cyclical process. For example, there was evidence that patients' past experiences of being uninvolved in consultations had contributed to a low expectation and desire for involvement. ${ }^{20,32,54}$ Similarly, the model postulates that the three factors identified outside the consultation influence each other. For example, patients who do not feel they possess the knowledge to contribute to decisions then lack desire or expectation for involvement.

When considering factors outside the consultation, there was evidence that involvement could be blocked by health professionals' attitudes, for example, a blanket view of patients' incapability. In discussing organisational challenges, professionals (with some agreement from carers) identified constraints in their day-to-day work, such as pressures from colleagues to prescribe certain drugs, which prevented patients' involvement. Although the development of consumer groups was raised as a contextual influence that has increased involvement, no other facilitators were raised. Further research into the nature of organisational barriers would be valuable in understanding how professionals could prioritise patient involvement above these constraints. For example, implementation theory suggests that 'transformational' leadership is important when implementing evidencebased practice. ${ }^{56}$ Patient perspectives revealed little awareness of the organisational barriers constraining health professionals. Although the barriers identified by health professionals seemed to be regarded as insurmountable, patient perspectives suggested that this was not always the case. Patients highlighted health professionals' failings to provide them with adequate information, which consequently had a detrimental effect on their perceived capability and confidence for involvement. Although there is a clear need to improve patients' knowledge, precisely how knowledge is best imparted is unclear, 
and the process is likely to be further hindered by the relational difficulties identified in the consultation.

There was evidence that some patients neither desired nor expected involvement, instead opting to entrust decisions to the professional. While it is important to respect that patients can make an informed choice not to be involved, it is likely that several factors encouraged passivity, such as past experiences of relatively paternalistic practice. One qualitative study involving people with chronic physical health conditions found that, compared with patients from high socioeconomic groups, those from lower socioeconomic groups were less likely to ask questions within consultations, believing it not to be within their role. ${ }^{57}$ This suggests that there are individual differences between patients, a subgroup of whom do not feel entitled to be involved. This underlines the need for health professionals to not take passivity at face value, but instead to help patients to establish, as Protheroe et al ${ }^{57}$ have discussed, 'permission to participate'.

The most prominent theme within the synthesis, central to the model, was the consultation setting and process factors within it. In the consultation setting, patients felt that their involvement was constrained by lack of time within the consultation, yet only one study involving health professionals identified time as a barrier. This suggests that prescribers fail to recognise that patients regard time as a barrier to their involvement. Although patients and prescribers understood the importance of their relationship and, in particular, the prescribers' responsibility in fostering a collaborative one, the majority of barriers came from the patient perspective. They identified the following relational factors as hindering their interactions: professionals' disregard of patients' opinions, feeling negatively perceived by the health professional, lack of trust and a pressure to appease the prescriber. Although all these factors were conceptualised as relational processes in the synthesis, it is important to acknowledge that interactions are based on relationships built over time. In addition, it should be acknowledged that interactions are likely to be influenced by broader influences, such as cultural perceptions of medicine and views of medical practitioners as powerful. $^{58}$

It is notable that the majority of facilitators identified within the evidence emerged within the consultation theme. There was evidence that involvement may be facilitated through the use of decision aids such as SDM computer packages, which increased disclosure and developed a more comprehensive understanding of patients' concerns. This stakeholder perception is supported by findings from a recent Cochrane review, which found evidence that decision aids led to a range of benefits for people with a range of health conditions, such as improved clinician-patient communication, greater alignment between patient values and their care choices, improved knowledge, and reduced passivity. ${ }^{59}$ While decision aids may increase involvement, the fact that such tools are needed to generate open and rich discussion also points to difficulties in the face-to-face interactions with the prescriber. Indeed, the behaviour of non-prescribing staff in trying to coach patients in asserting opinion and the need to bring advocates to prescribing meetings are indicative of failings in the patient-prescriber relationship. While prescribers may not be wholly responsible for these difficulties, further work is needed to improve the quality of this critical relationship and to overcome barriers such as the power imbalance between patients and prescribers. This highlights the importance of health professionals employing good communication skills, by showing a holistic interest in patients and through listening and responding. Stakeholders agreed that these were fundamental to involvement and, while some prescribers may already possess these skills, additional training may be beneficial. ${ }^{20}$

This synthesis demonstrates that patient involvement in antipsychotic prescribing decisions depends on a complex multifactorial process in which all elements respond to and influence one another. As such, patient involvement cannot be expected to spontaneously emerge within prescribing discussions without first ensuring that they are underpinned by the necessary foundations for a collaborative relationship. This includes there being an organisational context where patient involvement can be prioritised above working pressures, but also relates to the beliefs and attitudes of both patients and prescribers, e.g. patient passivity and perceptions of patients' capability.

We would argue that many of the belief and attitudinal barriers identified through this synthesis reflect differences between stakeholders in the understanding of the rights and responsibilities of each individual in the prescribing partnership. Without such a shared model, it is unreasonable to expect that patients will challenge and speak up in the determination of their own treatment, and that prescribers will challenge those obstacles that inhibit patient involvement. From the earliest points of engagement with services, patients need to understand that they have a right to involvement, which can only be removed in very specific circumstances. Additionally, both patients and prescribers need to understand that collaboration cannot be achieved without each partner being willing to value their own, as well as the other's, expertise. It may be that specific interventions could be adopted to support this understanding, for example, by educating patients on how their involvement may benefit their care and their satisfaction in interactions. Prescribers need to understand their responsibilities to foster opportunities for involvement wherever possible, by challenging barriers rather than conceding to them. This could involve explicit conversations between patient and prescriber about involvement to understand individuals' specific barriers. For example, patients who express a lack of interest in involvement should be asked why, to understand any barriers inhibiting their contribution and to determine steps to overcome these, such as information provision or simply giving them sufficient time for involvement.

Prescribing decisions involving people with SMI are influenced by a complex system of values, processes and constraints. The multifaceted and interconnected nature of these factors emphasises the need to take a global and holistic approach to enhancing SDM for antipsychotic treatments. Some parallels can be drawn with recent non-systematic work that has suggested that the involvement of people with mental health problems may depend upon factors beyond the nature of their individual interactions with prescribers. ${ }^{11}$ Our systematic approach to reviewing the literature has provided a comprehensive assessment of the evidence base and, importantly, enabled the integration of multiple stakeholder views. As such, it provides a broader view than any single reference. Our analysis is the first to identify differences in perspectives between patient and prescriber, which provides meaningful and contextually relevant opportunities for intervention.

\section{Strengths and limitations}

Our inclusion criteria allowed for the incorporation of studies without a specific aim to examine barriers and facilitators to patient involvement in antipsychotic prescribing, for example, those focusing on topics such as adherence and SDM more broadly. While this captured the breadth of findings in this field, it also led to some subjectivity in identifying those findings specific to involvement in prescribing decisions. To guard against this, two reviewers independently extracted all findings; where disagreements in interpretation arose, they were discussed to arrive at a final version to be used for the synthesis.

As part of our inclusion criteria, we operationalised any medication use in people with SMI as relating to antipsychotic medication, which may have had an impact on the studies included in our review. For example, Lester's study ${ }^{44}$ of views about the 
involvement of people with SMI in a primary care setting may refer to medications prescribed for other conditions in this population, such as physical health problems. However, this study's findings were deemed salient to the broader process of patient involvement in prescribing and were therefore retained.

The generalisability of our findings may also be limited by the characteristics of the primary studies included. All included studies originated from Western Europe or the USA/Canada. As such, the applicability of the findings and conclusions drawn may not translate to all international care settings. In addition, the greater proportion of studies conducted in community settings suggests that our findings may not readily apply to other settings, e.g. in-patient. Further primary research in a range of settings therefore seems warranted, as the particular barriers and facilitators to involving patients in prescribing are likely to differ.

We did not use any indicator of study quality to guide our synthesis. Although this is a limitation of this study, we would argue that our inclusion/exclusion criteria, which specified a minimum of a thematic type analysis, ensured that all studies provided a degree of 'richness'. It is also possible that relevant studies may have been excluded from our review owing to the authors' failure to report key characteristics of their sample. For example, some papers which included patients in in-patient settings were excluded owing to the sample being unclear; it is likely that a substantial number of participants within these settings would in fact meet the criteria. Future studies should ensure that their methods and findings are clearly described.

\section{Future directions}

Few studies incorporated family members' views on patient involvement in prescribing, although it is noteworthy that several studies not eligible for review included carers' views on their own involvement in medication decisions. Future studies may consider seeking the perspectives that family members hold about the patient's involvement, and the benefits or challenges this may present to carers, particularly as these individuals play a key part in the management of SMI.

Some of the themes contained very few facilitators; therefore, further research should aim to elicit such facilitators, particularly those that overcome the organisational barriers of patient capacity and passivity perceived by professionals. Further research is needed to empirically test whether the factors implicated within this model truly influence patients' involvement in medication decisions, and whether these factors interact in the way hypothesised. Finally, the findings suggest that future research needs to develop and test new interventions that break down patient barriers to involvement, not only at the consultation (e.g. through SDM tools), but also at a more fundamental level that challenges situational, attitudinal and belief-based barriers, e.g. helping to educate patients on the value of their knowledge and rights to involvement.

Rebecca Pedley, BSc, Division of Nursing, Midwifery and Social Work, School of Health Sciences, Faculty of Biology, Medicine and Health, The University of Manchester, Manchester Academic Health Science Centre, Room 6.306 Jean McFarlane Building, Oxford Road, Manchester, M13 9PL; Caitlin McWilliams, Division of Nursing, Midwifery and Social Work, School of Health Sciences, Faculty of Biology, Medicine and Health, The University of Manchester, Manchester Academic Health Science Centre, Oxford Road, Manchester, M13 9PL; Karina Lovell, PhD, Division of Nursing, Midwifery and Social Work, School of Health Sciences, Faculty of Biology, Medicine and Health, The University of Manchester, Manchester Academic Health Science Centre, Oxford Road, Manchester, M13 9PL; Helen Brooks, PhD, Department of Psychological Sciences, Institute of Psychology, Health and Society, University of Liverpool, Liverpool, L69 3BX: Kelly Rushton, PhD, Division of Nursing, Midwifery and Social Work, School of Health Sciences, Faculty of Biology, Medicine and Health, The University of Manchester, Manchester Academic Health Science Centre, Oxford Road, Manchester, M13 9PL; Richard J. Drake, MRCPsych, PhD, Division of Psychology and Mental Health, School of Health Sciences, Faculty of Biology, Medicine and Health, The University of Manchester, Manchester Academic Health Science Centre, Oxford Road, Manchester, M13 9PL; Barnaby Rumbold, MA, Millbrook Independent Hospital, Wythenshawe, Manchester, M23 2RX; Vicky Bell, BA, Division of Nursing, Midwifery and Social Work, School of Health
Sciences, Faculty of Biology, Medicine and Health, The University of Manchester, Manchester Academic Health Science Centre, Oxford Road, Manchester, M13 9PL; Penny Bee, PhD, Division of Nursing, Midwifery and Social Work, School of Health Sciences, Faculty of Biology, Medicine and Health, The University of Manchester, Manchester Academic Health Science Centre, Oxford Road, Manchester, M13 9PL.

Correspondence: Rebecca Pedley, Division of Nursing, Midwifery and Social Work, School of Health Sciences, Faculty of Biology, Medicine and Health, The University of Manchester, Manchester Academic Health Science Centre, Room 6.306 Jean McFarlane Building, Oxford Road, Manchester, M13 9PL.

Email: rebecca.pedley@manchester.ac.uk

First received 22 Sep 2017, final revision 26 Oct 2017, accepted 9 Nov 2017

\section{Author contributions}

R.P., P.B., K.L., H.B., K.R. and R.D. contributed to the design and conception of the study. All authors were involved in the screening of texts for inclusion in the review. R.P., C.M., V.B. and B.R. extracted the data. The analysis and interpretation of data was led by R.P. and C.M., with supervision from P.B., H.B. and K.L. All authors contributed to the analysis and interpretation of the synthesis. R.P. and C.M. led the write-up of the manuscript. All other authors provided critical review of the manuscript and assisted with revising the manuscript. All authors approved the final manuscript for submission.

\section{Funding}

This manuscript presents independent research (EQUIP) funded by the National Institute for Health Research (NIHR) under the Programme Grants for Applied Research programme (RPPG-1210-12007). The views expressed are those of the authors and not necessarily those of the National Health Service, the NIHR or the Department of Health.

\section{Acknowledgements}

Many thanks to Peter Coventry, who assisted with the design of the study and initial abstract screening. Many thanks to Owen Price, Amy Blakemore and Christine Molloy for their feedback on the manuscript.

\section{Supplementary material}

Supplementary material is available online at http://doi.org/10.1192/bjo.2017.5

\section{References}

1 Holman $\mathrm{H}$, Lorig $\mathrm{K}$. Patients as partners in managing chronic disease. Partnership is a prerequisite for effective and efficient health care. BMJ 2000; 320(7234): 526-7.

2 Department of Health. The Expert Patient: A New Approach to Chronic Disease Management for the 21st Century. Department of Health, London, 2001.

3 Patel SR, Bakken S, Ruland C. Recent advances in shared decision making for mental health. Curr Opin Psychiatry 2008; 21(6): 606-12.

4 Charles C, Gafni A, Whelan T. Shared decision-making in the medical encounter: what does it mean? (or it takes at least two to tango). Soc Sci Med 1997; 44(5): 681-92.

5 Slade M. Implementing shared decision making in routine mental health care. World Psychiatry 2017; 16(2): 146-53.

6 Department of Health. Liberating the NHS: No Decision About Me, Without MeGovernment Response to the Consultation. Department of Health London, 2012.

7 Frosch DL, Moulton BW, Wexler RM, Holmes-Rovner M, Volk RJ, Levin CA. Shared decision making in the United States: policy and implementation activity on multiple fronts. Zeitschrift für Evidenz, Fortbildung und Qualität im Gesundheitswesen 2011; 105(4): 305-12.

8 Gray R, Rofail D, Allen J, Newey T. A survey of patient satisfaction with and subjective experiences of treatment with antipsychotic medication. J Adv Nurs 2005; 52(1): 31-7.

9 Harris K, Brooks H, Lythgoe G, Bee P, Lovell K, Drake RJ. Exploring service users', carers' and professionals' perspectives and experiences of current antipsychotic prescribing: a qualitative study. Chronic IIIn 2017; 13(4): 275-87.

10 Adams JR, Drake RE, Wolford GL. Shared decision-making preferences of people with severe mental illness. Psychiatr Serv 2007; 58(9): 1219-21.

11 Morant N, Kaminskiy E, Ramon S. Shared decision making for psychiatric medication management: beyond the micro-social. Health Expect 2016; 19(5): 1002-14.

12 Hamann J, Leucht S, Kissling W. Shared decision making in psychiatry. (0001$690 \times($ Print)). 
13 National Institute for Clinical Excellence. Psychosis and Schizophrenia in Adults: Prevention and Management. National Clinical Practice Guidelines Number CG178: NICE, 2014.

14 Leucht S, Cipriani A, Spineli L, Mavridis D, Örey D, Richter F, et al. Comparative efficacy and tolerability of 15 antipsychotic drugs in schizophrenia: a multipletreatments meta-analysis. Lancet 2013; 382(9896): 951-62.

15 Lieberman JA, Stroup TS, McEvoy JP, Swartz MS, Rosenheck RA, Perkins DO, et al. Effectiveness of antipsychotic drugs in patients with chronic schizophrenia. N Engl J Med 2005; 353(12): 1209-23.

16 Jones PB, Barnes TE, Davies L, Dunn G, Lloyd H, Hayhurst KP, et al. Randomized controlled trial of the effect on quality of life of second- vs first-generation antipsychotic drugs in schizophrenia: cost utility of the latest antipsychotic drugs in schizophrenia study (CUtLASS 1). Arch Gen Psychiatry 2006; 63(10): 1079-87.

17 Tiihonen J, Walhbeck K, Lönnqvist J, Klaukka T, Ioannidis JPA, Volavka J, et al. Effectiveness of antipsychotic treatments in a nationwide cohort of patients in community care after first hospitalisation due to schizophrenia and schizoaffective disorder: observational follow-up study. BMJ 2006; 333(7561): 224.

18 Muench J, Hamer AM. Adverse effects of antipsychotic medications. Am Fam Physician 2010; 81(5): 617-22.

19 Duncan $E_{\text {, Best }} \mathrm{C}$, Hagen S. Shared decision making interventions for people with mental health conditions. Cochrane Database Syst Rev 2010; 2010(1): CD007297.

20 Mahone IH, Farrell S, Hinton I, Johnson R, Moody D, Rifkin K, et al. Shared decision making in mental health treatment: qualitative findings from stakeholde focus groups. Arch Psychiatr Nurs 2011; 25(6): e27-36.

21 Cochrane Handbook for Systematic Reviews of Interventions Version 5.1.0 [updated March 2011]. In: The Cochrane Collaboration (eds J Higgins, S Green): 2011

22 Tong $A$, Sainsbury $P$, Craig J. Consolidated criteria for reporting qualitative research (COREQ): a 32-item checklist for interviews and focus groups. Int J Qual Health Care 2007; 19(6): 349-57.

23 Mays N, Pope C. Assessing quality in qualitative research. BMJ 2000; 320 (7226): 50.

24 Thomas J, Harden A. Methods for the thematic synthesis of qualitative research in systematic reviews. BMC Med Res Methodol 2008; 8(1): 45

25 Barnett-Page E, Thomas J. Methods for the synthesis of qualitative research: a critical review. BMC Med Res Methodol 2009; 9(1): 59.

26 Britten N, Campbell R, Pope C, Donovan J, Morgan M, Pill R. Using meta ethnog raphy to synthesise qualitative research: a worked example. J Health Serv Res Policy 2002; 7(4): 209-15.

27 Besenius C, Bradley E, Nolan P. Attitudes of psychiatrists, nurses and service users towards prescribing and administrating depot antipsychotic medication. J Ment Health Train Educ Pract 2012; 7(1): 9-19.

28 Carrick R, Mitchell A, Powell RA, Lloyd $K$. The quest for well-being: a qualitative study of the experience of taking antipsychotic medication. Psycho Psychother 2004; 77(1): 19-33.

29 Chafetz L. The experience of severe mental illness: a life history approach. Arch Psychiatr Nurs 1996; 10(1): 24-31.

30 Chaplin R, Lelliott P, Quirk A, Seale C. Negotiating styles adopted by consultant psychiatrists when prescribing antipsychotics. Adv Psychiatr Treat 2007; 13(1): $43-50$.

31 Deegan PE, Rapp C, Holter M, Riefer M. A program to support shared decision making in an outpatient psychiatric medication clinic. Psychiatr Serv 2008; 59 (6): 603-5.

32 Delman J, Clark JA, Eisen SV, Parker VA. Facilitators and barriers to the active participation of clients with serious mental illnesses in medication decision making: the perceptions of young adult clients. J Behav Health Serv Res 2014; 42(2): 238-53.

33 Deslandes RE, John DN, Deslandes PN. An exploratory study of the patient experience of pharmacist supplementary prescribing in a secondary care mental health setting. Pharm Pract (Granada) 2015; 13(2): 553.

34 El-Mallakh P, Howard PB, Bond GR, Roque AP. Challenges of implementing a medication management evidence-based practice in a community mental health setting: results of a qualitative study. Issues Ment Health Nurs 2014; 35(7): 517-25.

35 Forchuk C, Jewell J, Tweedell D, Steinnagel L. Role changes experienced by clinical staff in relation to clients' recovery from psychosis. J Psychiatr Ment Health Nurs 2003; 10(3): 269-76.

36 Francis SA, Patel M. Caring for people with schizophrenia: family carers involvement with medication. Int J Pharm Pract 2000; 8(4): 314-23.

37 Gibson S, Brand SL, Burt S, Boden ZV, Benson O. Understanding treatment nonadherence in schizophrenia and bipolar disorder: a survey of what service users do and why. BMC Psychiatry 2013: 13: 153.
38 Goscha R, Rapp C. Exploring the experiences of client involvement in medication decisions using a shared decision making model: results of a qualitative study. Community Ment Health J 2015; 51: 267-74.

39 Green CA, Polen MR, Janoff SL, Castleton DK, Wisdom JP, Vuckovic N, et al. Understanding how clinician-patient relationships and relational continuity of care affect recovery from serious mental illness: STARS study results. Psychiatr Rehabil J 2008; 32(1): 9-22.

40 Hagen BF, Nixen G, Peters T. The greater of two evils? How people with transformative psychotic experiences view psychotropic medications. Ethical Hum Psychol Psychiatry 2010; 12(1): 44-59.

41 Hon A. Factors influencing the adherence of antipsychotic medication (Aripiprazole) in first-episode psychosis: findings from a grounded theory study. J Psychiatr Ment Health Nurs 2012; 19(4): 354-61.

42 lyer S, Banks N, Roy M-A, Tibbo P, Williams R, Manchanda R, et al. A qualitative study of experiences with and perceptions regarding long-acting injectable antipsychotics: part I-patient perspectives. Can J Psychiatry 2013; 58(5 suppl, 1): $14 \mathrm{~s}-22 \mathrm{~s}$.

43 Lacasse JR, Hayes Piel M, Lietz CA, Rider A, Hess JZ. The client experience of psychiatric medication: a qualitative study. Soc Work Ment Health 2016; 14 (1): $61-81$.

44 Lester $\mathrm{H}$, Tait L, England $\mathrm{E}$, Tritter J. Patient involvement in primary care mental health: a focus group study. Br J Gen Pract 2006; 56(527): 415-22.

45 Lorem GF, Frafjord JS, Steffensen M, Wang CE. Medication and participation: a qualitative study of patient experiences with antipsychotic drugs. Nurs Ethics 2014; 21(3): 347-58.

46 Malik A, Das AK. A qualitative study of the attitudes of patients in an early intervention service towards antipsychotic long-acting injections. Ther Adv Psychopharmacol 2014; 4(5): 179-85

47 Martean L, Evans C. Prescribing for personality disorder: qualitative study of interviews with general and forensic consultant psychiatrists. Psychiatr Bull 2014; 38(3): 116-21.

48 Mikesell L, Bromley E, Young AS, Vona P, Zima B. Integrating client and clinician perspectives on psychotropic medication decisions: Developing a Communication-centered Epistemic Model of Shared Decision Making for mental health contexts. Health Commun 2016; 31(6): 707-17.

49 Rogers A, Day J, Randall F, Bentall RP. Patients' understanding and participation in a trial designed to improve the management of anti-psychotic medication - a qualitative study. Soc Psychiatry Psychiatr Epidemiol 2003; 38 (12): $720-7$

50 Sajatovic M, Davies M, Bauer MS, McBride L, Hays RW, Safavi R, et al. Attitudes regarding the collaborative practice model and treatment adherence among individuals with bipolar disorder. Compr Psychiatry 2005; 46: 272-7.

51 Seale $C$, Chaplin R, Lelliott $P$, Quirk A. Sharing decisions in consultations involving anti-psychotic medication: a qualitative study of psychiatrists' experiences. Soc Sci Med 2006; 62(11): 2861-73.

52 Shepherd A, Shorthouse O, Gask L. Consultant psychiatrists' experiences of and attitudes towards shared decision making in antipsychotic prescribing, a qualitative study. BMC Psychiatry 2014; 14(1): 127.

53 Stewart DC, Anthony GB, Chesson R. 'It's not my job. I'm the patient not the doctor: patient perspectives on medicines management in the treatment of schizophrenia. Patient Educ Couns 2010; 78(2): 212-7.

54 Svedberg B, Lutzen K. Psychiatric nurses' attitudes towards patient autonomy in depot clinics. J Adv Nurs 2001; 35(4): 607-15.

55 Tranulis C, Goff D, Henderson DC, Freudenreich O. Becoming adherent to antipsychotics: a qualitative study of treatment-experienced schizophrenia patients. Psychiatr Serv 2011; 62(8): 888-92.

56 Rycroft-Malone J. The PARIHS framework - a framework for guiding the implementation of evidence-based practice. J Nurs Care Qual 2004; 19(4): 297-304.

57 Protheroe J, Brooks H, Chew-Graham C, Gardner C, Rogers A. 'Permission to participate?' A qualitative study of participation in patients from differing socio-economic backgrounds. J Health Psychol 2013; 18(8): 1046-55.

58 Nimmon L, Stenfors-Hayes T. The "Handling" of power in the physician-patien encounter: perceptions from experienced physicians. BMC Med Educ 2016; 16 (1): 114.

59 Stacey D, Légaré F, Lewis K, Barry MJ, Bennett CL, Eden KB, et al. Decision aids for people facing health treatment or screening decisions. Cochrane Database Syst Rev 2017; 2017(4): CD001431.
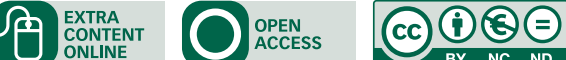